\title{
Capture-recapture method to assess the prevalence of disabled leprosy patients
}

\author{
JACQUES VAN DEN BROEK*, \\ THEO VAN JAARSVELD $* * *$ AD DE RIJK ${ }^{\dagger}$, \\ KEFAS SAMSON ${ }^{\dagger \dagger}$ \& PHILIP PATROBAS ${ }^{\dagger \dagger}$ \\ *Royal Tropical Institute (KIT), Wibautstraat 137j, 1090 DN \\ Amsterdam, The Netherlands \\ **Netherlands Leprosy Relief (NLR) (Present address Grevinckstraat \\ 26, 6525 CH Nijmegen, The Netherlands) \\ ${ }^{\dagger}$ Sophialaan 6, 1075 BR Amsterdam, The Netherlands \\ ${ }^{\dagger \dagger}$ Netherlands Leprosy Relief (NLR), Yelwa Club Room 3, PO Box \\ 759, Bukuru, Plateau State, Nigeria
}

Accepted for publication 22 May 2001

\begin{abstract}
Summary The capture-recapture technique was applied in estimating the prevalence of disabled leprosy patients in four States in Northern Nigeria. A two-sample capturerecapture method, using data from hospital admissions during 1997 and 1998 in three leprosy referral hospitals, and from a sample survey on leprosy patients with disabilities in the clinics in 1999. In the sample, 1395 (ex) leprosy patients were found, 393 with a disability. Of these 393 patients, 47 had been admitted during 1997 and 1998 to one of three leprosy referral hospitals. In these hospitals, 151 individuals from the 24 study Local Government Areas (LGA) in four states of Northern Nigeria were admitted in 1997 and 1998. Using the Peterson estimator, we calculated the number of unknown disabled leprosy patients in the studied LGAs to be 1262 (95\% confidence interval 991-1533). This was nearly four times greater than the field reported figure. The capture-recapture method can be applied in a leprosy care programme. Limitations of the method are the completeness of reporting after invitation in the field, as well as the probable biased sample of leprosy patients admitted to hospital. Our finding implies that relying on patients to report for prevention of disabilities and rehabilitation to the clinics, causes the real size of the problem to be underestimated by a factor of 3-4. We recommend the use of a special 'care' register for disabled leprosy patients to better address their needs for prevention of disabilities and rehabilitation.
\end{abstract}

\section{Introduction}

In any prevention of disabilities and rehabilitation programme for leprosy one would like to know the effective coverage of the services. The number of patients in need of care within a

Correspondence to: J. van den Broek (e-mail: j.v.d.broek@kit.nl) 
given country is often unknown. Care for disabled leprosy patients depends largely on the existence of a prevention of disabilities programme and of functioning leprosy referral hospitals with services for physical rehabilitation. Initially, leprosy control programmes originated from these leprosy hospitals. It is therefore unsurpising that traditionally, most leprosy patients would find their way to such hospitals, often circumventing the referral system of leprosy control programmes.

Even in the literature there are few reliable estimates of the disability burden from leprosy. However, the number of leprosy patients in need of care can be estimated by sampling or modelling.

1. Population surveys of persons with disabilities due to leprosy, like Rapid Village Surveys or Leprosy Elimination Campaigns, and extrapolation of the findings to the whole population.

2. Inviting all leprosy patients with a disability in the catchment area of a clinic to come forward, after selecting clinics in a sample survey.

3. Demographic modeling, ${ }^{1}$ based on age and sex specific incidence of impairments and disabilities, estimated life expectancy, and the dynamics of impairments and disabilities, both during and after treatment.

4. Capture-recapture techniques among leprosy patients with disabilities, registered at the referral hospital and recaptured during a field survey.

In this paper we discuss the application of methods 2 and 4.

Capture-recapture ${ }^{\mathrm{a}}$ methods were originally developed in wildlife conservation, ${ }^{2}$ and later applied in a variety of fields, notably in demography, epidemiology, criminology and service schemes for people with chronic conditions, such as cancer patients, ${ }^{3,4}$ homeless, alcoholics and other substance abusers, ${ }^{5-8}$ HIV positive people, ${ }^{9,10}$ and other marginalized and covert populations, ${ }^{11}$ including disabled persons. ${ }^{12}$ Many authors consider the method very useful in counting people in public health studies, ${ }^{13,14}$ the technique being quick, easy and cheap. Others have offered criticism, specifically concerning the issue of validation of the calculations ${ }^{15}$ and problems with perfect matching, ${ }^{16}$ the more so in developing countries.

The principle of capture-recapture is to use two or more overlapping sources of information. It measures prevalence of individuals based on two or more samples, whereby individuals are marked during the first sample and recaptured during one or more successive samples.

The ratio of individuals registered (marked) in both samples $(\mathrm{m})$ and patients registered in the first sample (M), equals the ratio of all individuals in the second sample (n) and the total unknown number $(\mathrm{N})$. This relationship is also known as the Peterson estimator, ${ }^{17}$ namely $\mathrm{m} / \mathrm{M}=\mathrm{n} / \mathrm{N}$ (Figure 1).

We conducted a sample survey of disabled leprosy patients reporting to clinics, proportional to the number of patients registered for MDT. This survey was carried out in six Local Government Areas (LGA) in each of four states of Northern Nigeria, as part of a needs assessment study. We included a capture-recapture method to assess its utility in estimating the prevalence of patients in need of care. We then compared this with the results of the sample survey, to assess patient compliance when invited to report to the clinic.

Over the past 7 years, the average leprosy case detection in Nigeria has been 7120 new

\footnotetext{
${ }^{\text {a }}$ The term capture-recapture may appear stigmatizing when used for humans. However, it is the commonly used term. Some authors use marking-recatpure, or dual record matching instead.
} 


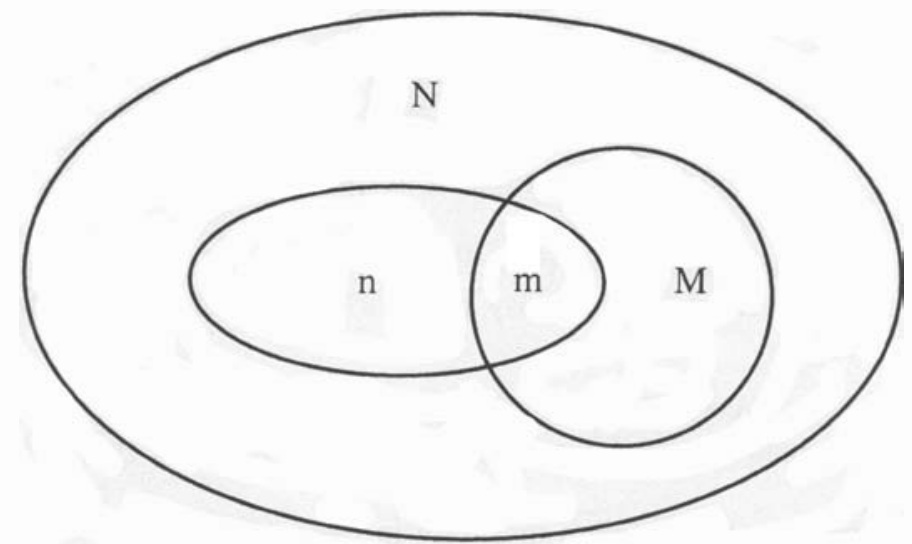

Figure 1. Graphical representation of the relationship between captured (n) and recaptured (M) populations, their overlap $(\mathrm{m})$, and the estimated total $(\mathrm{N})$ population.

cases per year, with on average $16 \%$ of these having a disability grade 2 according to the WHO disability scale.

In Northern Nigeria, there are 13 states supported by the Netherlands Leprosy Relief (NLR), 10 of which are serviced by a leprosy referral hospital (Figure 2). In these 13 states alone, over $55 \%$ of all new leprosy patients in Nigeria are diagnosed, while $37 \%(46,550,000$ out of $126,375,000$ ) of the population lives in these states.

Leprosy control programmes, including services for the prevention of disabilities and physical rehabilitation, have been functioning already for some time. Admission of leprosy patients in other than the leprosy referral hospitals rarely occurs.

\section{Materials and methods}

We selected four states out of the 13 NLR-supported states in Northern Nigeria: Benue, Borno, Gombe and Yobe States. These states were selected on operational grounds, such as capacity for research and non-involvement of the programme staff in other concurrent important activities. In 1998, there were 1469 leprosy patients registered for MDT. In each of these four states we selected six LGAs according to a sampling scheme, proportionate to size of registered leprosy patients, as was used for Rapid Village Surveys of leprosy in Thailand. ${ }^{18}$ We listed the LGAs with the number of patients registered for MDT, and divided the total number of patients by six to determine the sampling interval. We selected the first LGA with a dice and then continued to select the other five LGAs with the calculated interval. In the clinics of selected LGAs, there were 809 patients registered for MDT in 1998. Therefore, the total weighted sampling fraction was $0 \cdot 551$.

This study was part of an 'Assessment of Impairments and Needs' study to answer the questions of how many patients have disabilities, what kind and degree of disabilities they have, and what their needs for intervention are. For this purpose an instrument was developed and field tested, consisting of a questionnaire and a standardized checklist for recording results of physical examination. The questioning and physical examination was carried out by the State Leprosy Supervisor, under supervision of the State Leprosy Control Officer and/or the Prevention of Disability Supervisor. 


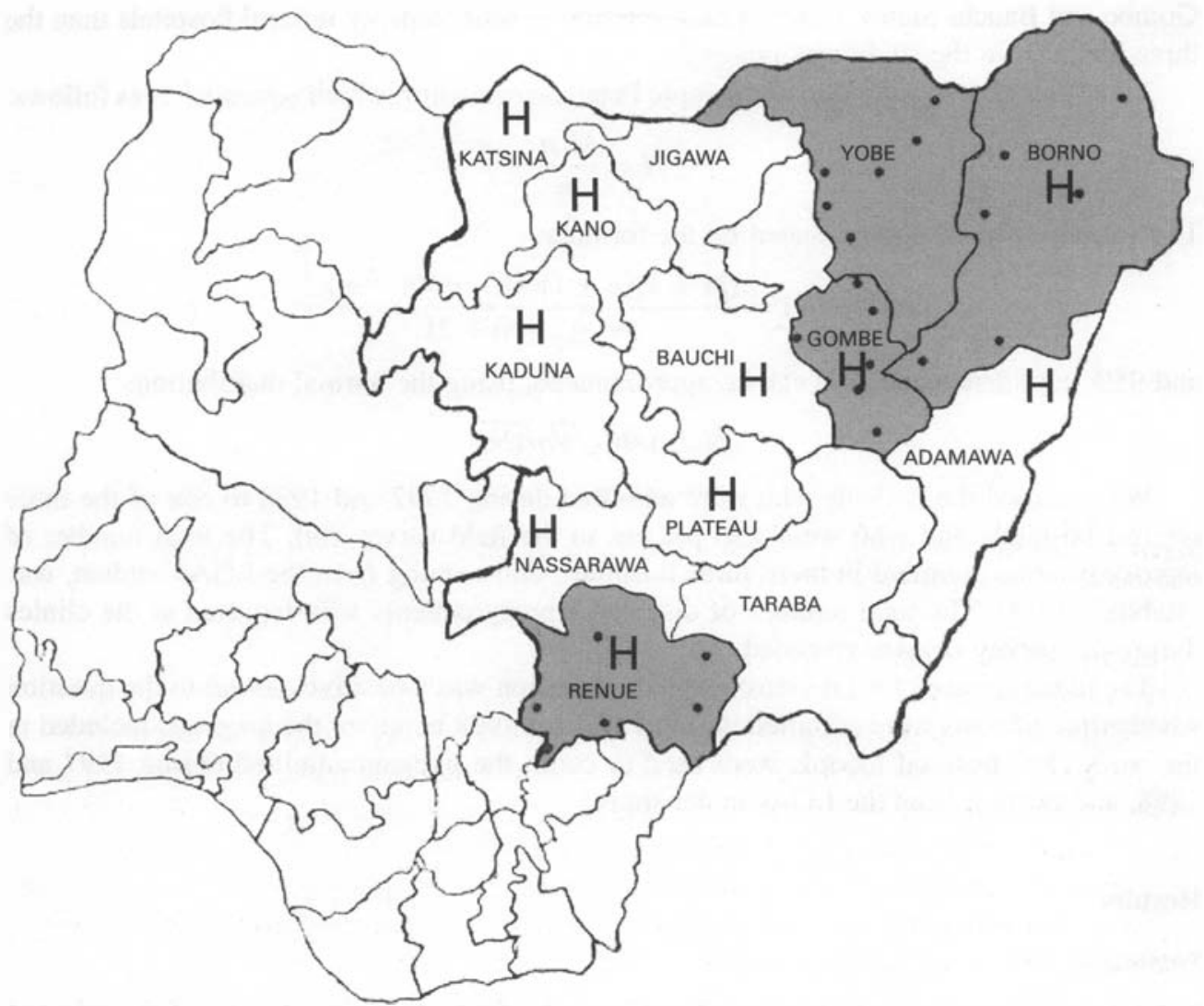

Key:

Named states: $\quad$ states supported by Netherlands Leprosy Relief.

Grayed states: $\quad$ states selected for study.

H: leprosy referral hospitals.

Dots: capitals of Local Government Area in which study was performed.

Figure 2. Map of Nigeria.

During monthly routine clinic days, announcements were made to all currently attending patients and ex-patients, requesting them, and any other ex-leprosy patients they knew, to attend the next monthly clinic. The community was further sensitized and mobilized through health staff, community leaders and leprosy chiefs.

At the next monthly clinic, all persons who came forward with a disability due to leprosy were interviewed and examined according to the standardized questionnaire and checklist. The WHO definition and grading of disability for leprosy was used.

We collected data from the three leprosy referral hospitals of the four states, namely Molai Hospital for Borno and Yobe States, Mkar Hospital for Benue and Bayara Hospital for 
Gombe and Bauchi States. Referral or admission to other leprosy referral hospitals than the three included in the study was ignored.

In the analysis, we used the two sample Peterson estimator, which equation ${ }^{\mathrm{b}}$ is as follows:

$$
N=\frac{M^{*} n}{m}
$$

The variance can be approximated by the formula:

$$
\operatorname{Var}(N)=\frac{(M+1)(n+1)(M-m)(n-m)}{(m+1)^{2}(m+2)}
$$

and $95 \%$ confidence intervals can be approximated, using the normal distribution:

$$
N \pm 1.96 \sqrt{\operatorname{Var}(N)}
$$

We recorded the patients who were admitted during 1997 and 1998 to one of the three referral hospitals and who were also present in the field survey $(m)$. The total number of leprosy patients admitted in these three hospitals, and coming from the LGAs studied, was established $(M)$. The total number of disabled leprosy patients who reported at the clinics during the survey $(n)$ was recorded.

The identifier used for previous hospital admission was a positive answer to the question whether the patients were admitted during 1997 and 1998 in any of the hospitals included in the study. The hospital records were used to count the patients admitted during 1997 and 1998, and coming from the LGAs under study.

\section{Results}

SAMPLE

A total of 1395 patients were included in the study, from all leprosy clinics of the selected 24 LGAs in four states. All were interviewed and physically examined. Of these, 393 had a disability or were experiencing leprosy reactions. Out of these patients, $81(21 \%)$ were still on MDT. On average, there were 15.7 disabled persons per LGA, with a standard error of $3 \cdot 7$.

With these data we could calculate the total number of person with disabilities due to leprosy in the four states by dividing 393 with the sampling fraction 0.551 , resulting in 714 , with a $95 \%$ confidence interval of 384-1043.

\section{CAPTURE-RECAPTURE METHOD}

Of the 393 patients $(\mathrm{n})$ found with disabilities, $47(\mathrm{~m})$ could be identified as also having been admitted during 1997 and 1998 to one of the three leprosy referral hospitals studied. In these hospitals, we identified 151 individuals (M) who were admitted in 1997 and 1998, coming from the 24 study LGAs.

Applying the Peterson estimator, we calculated the estimated number of disabled leprosy patients in the LGAs of the four states to be 1262 , with a $95 \%$ confidence interval from 991 to 1533 (Table 1).

\footnotetext{
${ }^{\mathrm{b}}$ Other authors recommend the use of Seber's adjustment to the unbiased Peterson estimator, ${ }^{4}$ which is suitable for smaller sample sizes and smaller overlapping registrations, and corrects for the fact that there is no replacement after recapture. Its equation is $N=\frac{(M+1)^{*}(n+1)}{m+1}$
} 
Table 1. Presence of patients with disability, admitted during 1997 and 1998, from hospital records and from field sample records, in the studied LGAs of four states combined. Between brackets are the parameters used in the formula. The findings from the study are presented in boldface

\begin{tabular}{lcccc}
\hline \multirow{2}{*}{ Presence of patients with disability } & \multicolumn{2}{c}{ Field data } \\
& & Yes & No & Total \\
\hline Hospital data & Yes & $47(\mathrm{~m})$ & 104 & $151(\mathrm{M})$ \\
& No & 346 & 765 & 1111 \\
& Total & $393(\mathrm{n})$ & 869 & $1262(\mathrm{~N})$ \\
& & & & \\
\hline
\end{tabular}

Table 2. Peterson's estimator and $95 \%$ confidence intervals of the number of leprosy patients with disabilities in the studied LGAs, stratified per state

\begin{tabular}{lrrrrrr}
\hline State & m & M & n & N & $95 \%$ CI & \\
\hline Benue State & 17 & 48 & 120 & 338 & 229 & 447 \\
Borno State & 13 & 61 & 102 & 478 & 289 & 667 \\
Gombe State & 12 & 23 & 44 & 84 & 59 & 109 \\
Yobe State & 5 & 19 & 127 & 482 & 223 & 741 \\
Total & 47 & 151 & 393 & 1382 & & \\
\hline
\end{tabular}

Stratification according to state yielded a similar overall estimate of 1382, although, of course, with relatively larger confidence intervals (Table 2).

We compared the age distribution of these categories with the total number of patients with disabilities found in the field, broken down to patients still on MDT at the time of the study and patients released from treatment (RFT) sometime in the past (Table 3). We did not further analyse patients without disabilities.

In addition, the disability grading, according to the summation of the WHO scores for eyes, hands and feet, both right and left, (also called the EHF score, with a range from 0 till 12) differed between these categories. 67\% (54 out of 81) of patients still on MDT had an EHF score of 4 or less, against $44 \%$ (138 out of 312) of RFT patients.

Additional analysis of the data shows that the difference in age distribution of the 151 admitted patients, as compared to the 47 recaptured, was statistically significant (Table 4 ).

Table 3. Age groups of MDT and RFT (released from treatment) patients with disabilities found in the field

\begin{tabular}{lcrrr}
\hline Age group & $\begin{array}{c}\text { MDT patients } \\
\text { with disabilities }\end{array}$ & $\%$ & $\begin{array}{c}\text { RFT patients } \\
\text { with disabilities }\end{array}$ & $\%$ \\
\hline $0-14$ years & 2 & 2 & 0 & \\
15-44 years & 44 & 54 & 86 & 69 \\
$>45$ years & 35 & 43 & 217 & 3 \\
Unkown & 0 & 100 & 312 & 100 \\
Total & 81 & & & \\
\hline
\end{tabular}


Table 4. Age groups of admitted and recaptured patients with disabilities

\begin{tabular}{lcrrr}
\hline Age group & $\begin{array}{c}\text { Admitted } \\
\text { patients }\end{array}$ & $\%$ & $\begin{array}{c}\text { Re-captured } \\
\text { patients }\end{array}$ & $\%$ \\
\hline 0-14 years & 5 & 3 & 1 & 2 \\
15-44 years & 82 & 54 & 14 & 30 \\
$>45$ years & 60 & 40 & 32 & 68 \\
Unknown & 4 & 3 & 0 & 100 \\
Total & 151 & 100 & 47 & \\
\hline
\end{tabular}

Chi square for trend $=6.468, P=0.011$.

We recaptured the older of the previously admitted patients. The records of the admitted patients did not allow a classification and analysis according to the EHF score.

In all, $223(57 \%)$ out of the 393 individuals found with a disability had, at the time of examination and in the opinion of the examiner, one or more indications for admission to a leprosy referral hospital. The indications included severe leprosy reaction (20 times), and/or septic or reconstructive surgery (201 times), and/or need for amputation and/or prosthesis (17 times).

\section{Discussion}

The estimate of 1262 (95\% confidence interval 991-1533) patients by applying the capturerecapture technique is a factor 3-4 higher than the 393 disabled leprosy patients found in the survey. The large unknown or hidden population of disabled leprosy patients could partly be explained by the fact that the registration of disabled patients at the clinic was a one-time event, rather than an ongoing process. Presently, no care register exists for disabled leprosy patients in Nigeria.

\section{ASSUMPTIONS UNDERLYING THE TECHNIQUE}

As a quick and cheap method, the two-sample capture-recapture method is an appropriate tool to gain an impression of the number of persons missed by the health care system. The confidence interval tends to be larger when the number of overlapping registrations $(\mathrm{m})$ are smaller. In our study the confidence interval of the grouped analysis did not exceed $22 \%$ of the calculated value.

However, the technique is rough and biased. Underlying assumptions and possible violations include the following:

- The population is closed (geographically and demographically), so that the size is constant. Disabled leprosy patients, like all human beings, tend to have complex and hidden patterns of behaviour, sometimes favouring distant instead of nearby hospitals. Although it is possible that some may have traveled to other states, this number is likely to be very small, given the huge distances to other leprosy referral hospitals and the existence of similar services nearby. Likewise, some patients may have died or migrated, and others may have developed new disabilities. However, this effect is likely to be 
minimal, given the expected small numbers of new disabled patients or death/resettlement over a period of 2 years.

- Homogeneity of the population, meaning that all individuals have the same chance of being hospitalized (captured or marked) and of subsequently being included in the sample (recaptured). The invitation procedure may have caused bias in the number and type of patients with disabilities who came forward. For instance, the inclusion of patients from leprosy settlements could present such a problem. These patients may have different demands for care, than disabled persons living in a normal village. There was one leprosy settlement under the 24 LGAs of this study, contributing only 22 disabled leprosy patients. Patients with previous admission could have a different health seeking behaviour and expectation of cure or care. A way to deal with heterogeneity would be stratified analysis. Stratification by state in our study revealed similar results to those without stratification (Table 2). Stratification by age revealed differences in the patients admitted and recaptured in the field. Stratification to 'on MDT' or 'RFT' was not possible, because these data were lacking in most of the hospital records.

- Independence assumption, meaning that admission (capture or marking) does not affect the chance of inclusion in the sample (recapture). The interval between the field study and the analysis of hospital records was 8 months. This allowed previously admitted patients to have returned to their home, enabling them to partcipate in the field survey. The choice of clinics where leprosy services are rendered is not random, but is related to the current caseload of patients requiring MDT. The accessibility of these clinics may not be adequate for all ex-patients in need of care, and may differ depending on geographical conditions, severity of the disability and varying attidues of health staff. These variations could not be further assessed. However, we have no reason to believe that geographical accessibility was related to the severity of disability.

- Perfect matching of individuals identified during admission to hospital and the the fields sample. Matching by soliciting a history of admission to the state leprosy referral hospital during the previous 2 years was considered sufficient to serve the purpose of application of the capture-recapture method. Possible recall bias of events such as hospital admission is unlikely, although the exact dates and duration of admission may be less acurately remembered. We did not use other identifiers, because addresses and even names may differ in time, and many patients do not recall their exact age.

\section{STUDY METHODOLOGY}

There may have been sampling errors in the field, related to different announcement strategies and impact. The willingness of leprosy victims to come forward to peripheral clinics may have differed. These factors are crucial factor for underlying assumptions of randomness and representativeness required for this method. In addition, the need felt by patients for admission to hospitals may vary over time, depending on changing physical conditions. An important factor hampering timely admission is the accessibility and affordability of services in terms of funds for transport and opportunity costs. The different age distribution between patients found in the field and admitted in the hospitals points to the fact that the younger people in particular were reluctant to come forward.

In addition, this study revealed that many patients had indications for referral to hospital. These patients were unknown to the health services. In general, the dynamics of leprosy patients reaching leprosy hospital, with and without referral, is still ill understood. 
It is a fact that patients often reach leprosy referral hospitals without being officially referred.

In a study in Tanzania, using a demographic model, ${ }^{1}$ the number of disabled patients predicted was four times greater than actually found in the clinics. This model was based on known age and sex specific disability incidence, estimated life expectancy of patients with and without disability, and the dynamics of disability state during and after treatment. This is a remarkably similar result. However, we have no means to prove that these results represent the reality. For that purpose, only a comparison with a whole population survey would be suitable.

\section{CONCLUSION}

The capture-recapture technique, in combination with a sample survey, appears to be a straightforward procedure to estimate the hidden population of disabled leprosy patients in need of care, and to assess the efficiency of a prevention of disabilities and rehabilitation programme. Comparison with demographic modeling revealed a remarkable consistency in the estimate.

We conclude that inviting disabled leprosy patients to come forward results in the capture of only a proportion of the actual cases in need of care. Obviously, the yield of invitation very much depends on the efficacy to convey the message and the willingness to come forward. Our sample therefore cannot be considered representative of the real number of leprosy patients with disabilities.

The actual coverage of clinics for MDT is likely to be different than that required for prevention of disabilities and rehabilitation. However, the patients who do report are likely to be motivated to participate in the prevention of disabilities and rehabilitation services.

We recommend that all disabled leprosy patients be registered in a special 'care' register, in order to enable their needs to be properly addressed.

\section{Acknowledgements}

We want to thank NLR (Netherlands Leprosy Relief) who made it possible to carry out this study. Of course, this study would not have been possible without the enormous help of the State Control Officers and State Supervisors of the four states in Nigeria where the study was carried out: Dr S. O. Ogiri (Benue State), Dr L. A. Mshelia (Borno State), Dr G. A. Manasa (Gombe State) and Dr A. Abdulwahab (Yobe State). Finally, we thank the NLR Representative in Nigeria, Mr Henk Plomp, who kindly facilitated the execution of this study.

\section{References}

${ }^{1}$ van den Broek J, van der Steen, Rubona J et al. Modeling the prevalence of persons affected by leprosy in Tanzania. Poster number EP59, Beijing World Leprosy Congress, 1998.

2 Seber GAF. The estimation of animal abundance and related parameters. London: Charles Griffin, 1982. ISBN 0-85264-262-8.

3 Robles SC, Marrett LD, Clarke EA, Risch HA. An application of capture-recapture methods to the estimation of completeness of cancer registration. J Clin Epidemiol, 1988; 41: 495-501.

4 Schouten LJ, Straatman H, Kiemeney LALM et al. The capture-recapture method for estimation of cancer registry completeness. Int J Epidemiol, 1994; 23: 1111-1116. 
${ }^{5}$ Larson A, Stevens A, Wardlaw G. Indirect estimates of hidden populations: capture-recapture methods to estimate the number of heroin users in the Australian Capital Territory. Soc Sci Med, 1994; 39: 823-831.

${ }^{6}$ Squires NF, Beeching NJ, Schlecht BJM, Ruben SM. An estimate of the prevalence of drug misuse in Liverpool and a spatial analysis of known addition. J Public Health Med, 1995; 17: 103-109.

7 Hay G, McKeganey N. Estimating the prevalence of drug misuse in Dundee, Scotland: an application of capturerecapture methods. J Epidemiol Commun Health, 1996; 50: 469-472.

${ }^{8}$ Hartnoll R, Lewis R, Mitcheson M, Bryer S. Estimating the prevalence of drug addiction. Lancet, 1995; 1: 203205.

9 Mastro TD, Kitayaporn D, Weniger BG et al. Estimating the number of HIV-infected injection drug users in Bangkok: a capture-recapture method. Am J Public Health, 1994; 84: 1094-1099.

10 McKeganey N, Barnard M, Leyland A et al. Female streetworking prostitution and HIV infection in Glasgow. $B M J, 1992 ;$ 30: 801-804.

${ }^{11}$ Fisher N, Turner SW, Plugh R, Taylor C. Estimating numbers of homeless and homeless mentally ill people in north east Westminster by using capture-recapture analysis. BMJ, 1994; 308: 27-30.

12 Gutteridge W, Collin C. Capture-recapture techniques. Quick and cheap. BMJ, 1994; 308: 531.

13 LaPorte R. Assessing the human condition: capture-recapture techniques. BMJ, 1994; 308: 5-6.

14 Watts CH, Zwi AB, Foster G. How to do (or not to do)... Using capture-recapture in promoting public health. Health Policy Planning, 1995; 10: 198-203.

15 Waters WE. More unreliable in humans than birds. $B M J, 1994 ; 308: 531$.

16 Black JFP, McLarty D. Difficult to use in developing countries. BMJ, 1994; 308: 531.

17 Bishop YMM, Fienberg EF, Holland PW. Discrete multivariate analysis: theory and practice. MIT Press, Cambridge, Massachusetts, 1975, ISBN 0-262-02113-7.

18 Pinitsoontorn S, Schreuder PAM, Chirawatkul A et al. Rapid Village Survey to determine the size of the leprosy problem in Khon Kaen Province, Thailand. Int J Lepr, 1996; 64: 51-57. 